OPEN ACCESS

Edited by:

Tae-Hee Cho,

Hospices Civils de Lyon, France

Reviewed by:

Jose Gutierrez,

Columbia University, United States

Linxin Li,

University of Oxford, United Kingdom

*Correspondence:

Min Lou

Im99@zju.edu.cn

Specialty section:

This article was submitted to Stroke,

a section of the journal

Frontiers in Neurology

Received: 08 November 2017

Accepted: 22 January 2018

Published: 08 February 2018

Citation:

Liu C, Shi F, Chen Z, Yan S, Ding X and Lou M (2018) Severe Blood-

Brain Barrier Disruption in

Cardioembolic Stroke.

Front. Neurol. 9:55.

doi: 10.3389/fneur.2018.00055

\section{Severe Blood-Brain Barrier Disruption in Cardioembolic Stroke}

\author{
Chang Liu', Feina Shi', Zhicai Chen ${ }^{1}$, Shenqiang Yan ${ }^{1}$, Xinfa Ding ${ }^{2}$ and Min Lou ${ }^{1 *}$ \\ ${ }^{1}$ Department of Neurology, School of Medicine, The 2nd Affiliated Hospital of Zhejiang University, Hangzhou, China, \\ ${ }^{2}$ Department of Radiology, School of Medicine, The 2nd Affiliated Hospital of Zhejiang University, Hangzhou, China
}

Background: Previous studies demonstrated that cardioembolism (CE) was prone to develop hemorrhagic transformation $(\mathrm{HT})$, whereas hyper-permeability of blood-brain barrier (BBB) might be one reason for the development of HT. We, thus, aimed to investigate whether the BBB permeability (BBBP) was higher in CE stroke than other stroke subtypes in acute ischemic stroke (AIS) patients.

Methods: This study was a retrospective review of prospectively collected clinical and imaging database of AIS patients who underwent CT perfusion. Hypoperfusion was defined as $\operatorname{Tmax}>6 \mathrm{~s}$. The average relative permeability-surface area product ( $\mathrm{rPS}$ ), reflecting the BBBP, was calculated within the hypoperfusion region (rPS hypo $_{\text {). }}$ CE was diagnosed according to the international Trial of Org 10172 in Acute Stroke Treatment criteria. Receiver operating characteristics (ROC) curve analysis was used to determine predictive value of $r P S_{\text {hypo }}$ for CE. Logistic regression was used to identify independent predictors for CE.

Results: A total of 187 patients were included in the final analysis [median age, 73 (61-80) years; 75 (40.1\%) females; median baseline National Institutes of Health Stroke Scale score, 12 (7-16)]. Median rPS hypo was 65.5 (35.8-110.1)\%. Ninety-seven (51.9\%) patients were diagnosed as $\mathrm{CE}$. ROC analysis revealed that the optimal rPS hypo $_{\text {threshold }}$ for CE was $86.71 \%$. The value of $r P S_{\text {hypo }}$ and the rate of $r P S_{\text {hypo }}>86.71 \%$ were significantly higher in patients with CE than other stroke subtypes $(p<0.05)$, after adjusting for the potential confounds.

Conclusion: The extent of BBB disruption is more severe in CE stroke than other stroke subtypes during the hyperacute stage.

Keywords: acute ischemic stroke, blood-brain barrier, cardioembolism, CT perfusion, stroke subtypes

\section{INTRODUCTION}

Cardioembolism (CE) is one of the major causes of ischemic stroke and its outcome is usually worse than other stroke subtypes (1). Studies have shown hemorrhagic transformation (HT), a potentially life-threatening complication of stroke, was more common in $\mathrm{CE}(2,3)$. Considering that the underlying mechanism of HT was the hyper-permeability, even damage of blood-brain barrier (BBB) $(4,5)$, we hypothesized that $\mathrm{CE}$ might associate with higher BBB permeability (BBBP) than other stroke subtypes.

CT perfusion (CTP) is a modern dynamic imaging technique that can evaluate BBBP by generating permeability-surface area product (PS) maps. Most studies focused on the relationship between BBBP and HT, while few explored the association between BBBP and stroke subtypes. Therefore, we 
aimed to assess the association between BBBP and CE, especially $\mathrm{BBBP}$ in the hypoperfusion region where most HT occurs.

\section{MATERIALS AND METHODS}

\section{Patients}

We reviewed our consecutively collected patients with acute ischemic stroke who received intravenous thrombolysis (IVT) between July 2011 to January 2016. We then enrolled patients who (1) underwent CTP before IVT suggesting acute anterior circulation stroke; (2) baseline hypoperfusion (defined as Tmax $>6 \mathrm{~s}$ ) volume $>10 \mathrm{ml}$. Exclusion criteria: (1) image quality was insufficient for analysis; (2) bilateral ischemic stroke. Intravenous alteplase was administered according to the international guidelines $(0.9 \mathrm{mg} / \mathrm{kg}, 90 \mathrm{mg}$ dose at maximum, $10 \%$ in a bolus in $1 \mathrm{~min}$ with the remaining dose in a 60-min infusion) (6).

We retrieved demographic, clinical, and laboratory data including age, sex, smoking history, comorbid conditions, such as history of hypertension, diabetes, hyperlipidemia, and atrial fibrillation; National Institutes of Health Stroke Scale (NIHSS) score, and onset-to-imaging time (OIT).

Stroke etiology was classified according to the Trial of Org 10172 in Acute Stroke Treatment (TOAST) criteria: large-artery atherosclerosis (LAA), cardioembolism (CE), small-artery occlusion (SAO), acute stroke of other determined etiology (ODE), and stroke of undetermined etiology (UDE) (7). Patients were dichotomized into $\mathrm{CE}$ group versus non-CE group.

\section{Imaging Protocols}

CT perfusion was performed on a $2 \times 64$-slice CT scanner (SOMATOM Definition Flash; Siemens Healthcare Sector, Forchheim, Germany), including non-enhanced head CT scan $(120 \mathrm{kV}, 320 \mathrm{~mA}$, effective dose $=2.19 \mathrm{mSv}$, contiguous $5 \mathrm{~mm}$ axial slices), and volume perfusion CT (VPCT) (4 s delay after start of contrast medium injection, $74.5 \mathrm{~s}$ total imaging duration, $80 \mathrm{kV}, 120 \mathrm{~mA}$, effective dose $=3.68 \mathrm{mSv}$ ). VPCT consisted of 26 consecutive spiral acquisitions of the brain from the base to the top of the skull, and were divided into four parts: (1) 2 scans with 3 s cycle time; (2) 15 scans with 1.5 s cycle time; (3) 4 scans with 3 s cycle time; and (4) 5 scans with 6 s cycle time. A 60 -ml bolus of non-ionic contrast medium (Iopamidol; $370 \mathrm{mg} / \mathrm{ml}$; Bracco Sine, Shanghai, China) was used at a flow rate of $6 \mathrm{ml} / \mathrm{s}$, followed by a $20 \mathrm{ml}$ saline chaser at $6 \mathrm{ml} / \mathrm{s}$.

\section{Image Analysis}

Cerebral blood flow (CBF) and Tmax were automatically calculated from CTP data with commercially available software (MIStar, Apollo Medical Imaging Technology, Melbourne, VIC, Australia). CTP-derived PS color maps were also automatically generated by MIStar permeability model based on the adiabatic approximation of tissue homogeneity model (8-10). A threshold of Tmax $>6 \mathrm{~s}$ was used for volumetric measurement of baseline hypoperfusion areas (11). Baseline relative CBF $<30 \%$ was used for calculating infarct volume (12). The baseline mismatch ratio was defined as the volume of hypoperfusion divided by the corresponding infarct volume (13).

Baseline hypoperfusion regions in the ischemic hemisphere were automatically outlined by MIStar on Tmax maps as regions of interest (ROI)s in each slice separately. These ROIs were manually checked and corrected by an experienced neuro-radiologist (Xinfa Ding) and two trained observers (Chang Liu and Feina Shi) who were blinded to other study data. We then superimposed these ROIs on the PS maps and CBF maps by each slice (thickness $=10 \mathrm{~mm}$ ), respectively, to calculate mean PS and CBF values within all ROIs. The mean PS and CBF were measured by squared voxels. PS represents the rate of iodinated contrast extravasation from the intravascular to the extravascular space through a damaged $\mathrm{BBB}$, and is influenced by $\mathrm{CBF}$ at the condition of ischemia. Therefore, it is more appropriate to use relative permeability-surface area product $(\mathrm{rPS}, \mathrm{rPS}=\mathrm{PS} / \mathrm{CBF} \times 100 \%)$ to represent $\mathrm{BBBP}$ in stroke patients $(14,15)$. The CBF and $\mathrm{rPS}$ of the hypoperfusion region were represented as $\mathrm{CBF}_{\text {hypo }}$ and $\mathrm{rPS}_{\text {hypo }}$, respectively.

Hemorrhagic transformation was defined as presence of hemorrhagic infarction ( $\mathrm{HI}$ ) or parenchymal hematoma $(\mathrm{PH})$ at $24 \mathrm{~h}$ after treatment according to European Cooperative Acute Stroke Study criteria (16).

\section{Statistical Analysis}

Median (25th-75th percentile) for non-normal distributed continuous variables and frequency (percentage) for categorical variables were used. Receiver operating characteristics (ROC) curve analysis was used to determine predictive value of $\mathrm{rPS}_{\text {hypo }}$ for CE. Chi-square test was used to compare the dichotomous variables between two groups, while independent samples Mann-Whitney $U$ test was used for non-normal distributed continuous variables. We performed multivariable logistic regression analysis to assess independent predictors of CE. Results are reported as odds ratios with $95 \%$ confidence intervals (CIs). A significance level of 0.05 was used to assess statistical difference. All statistical analyses were performed using SPSS Version 19.0 (IBM, Armonk, NY, USA).

\section{RESULTS}

Of 230 patients who met inclusion criteria, 43 patients were excluded [poor image quality due to excessive motion during imaging and trouble in contrast injection which affected measurement of PS $(n=24)$ and diagnosis of bilateral ischemic stroke $(n=19)]$. A total of 187 remaining patients were included for the final analysis, of which 158 patients underwent IVT alone and 29 patients had IVT combined with endovascular therapy. The median age was $73(61-80)$ years, and 75 (40.1\%) were female. Median baseline NIHSS score was 12 (7-16), and median OIT was 163 (96-240) min. rPS $_{\text {hypo }}$ ranged from $4.2-321.4 \%$ and its median value was $65.5(35.8-110.1) \%$. Demographic and clinical characteristics of patients included in and excluded from the current study were comparable (Table S1 in Supplementary Material). 
According to TOAST criteria, 97 (51.9\%) patients were diagnosed as CE, of whom 16 patients did not have atrial fibrillation: 2 had mechanical prosthetic valves, 3 had dilated cardiomyopathy, 1 had mitral annulus calcification, 9 had congestive heart failure, 1 had myocardial infarction. Numbers of patients diagnosed as LAA, SAO, ODE, and UDE were 43 (23.0\%), 0. $1(0.5 \%)$ and $46(24.6 \%)$, respectively. 14 patients were diagnosed as non-CE-presenting atrial fibrillation, among whom 11 patients were classified as A1-S0-C1-O0-D0, and 3 patients were classified as A1-S3-C1-O0-D0 according to ASCOD classification (17).

Receiver operating characteristics analysis revealed an optimal $\mathrm{rPS}_{\text {hypo }}$ threshold for $\mathrm{CE}$ of $86.71 \%$, with an area under the curve of 0.642 (95\% CI $0.563-0.721, p=0.001)$, and this yielded a sensitivity of $44.3 \%$ and a specificity of $80.0 \%$. As showed in Table 1, patients in CE group were older, and had higher female predominance and lower rate of current smokers than non-CE group (all $p<0.05$ ). The platelet counts and leukocyte counts were lower in CE group (both $p<0.05$ ). Baseline NIHSS, infarct volume and hypoperfusion volume were comparable between groups, while baseline mismatch ratio was significant lower in CE group $(p=0.033)$. Compared with non-CE group, $\mathrm{CE}$ group had lower $\mathrm{CBF}_{\text {hypo }}(p=0.032)$ and greater $\mathrm{rPS}_{\text {hypo }}(p=0.001)$. CE group had higher rate of HT than non-CE group $(p=0.038)$, while the rates of $\mathrm{HI}$ and $\mathrm{PH}$ were comparable.

Since atrial fibrillation was highly correlated with CE, it was not selected for adjustment in the binary logistic regression. Table 2 shows the results of multivariable logistic regression

TABLE 1 | Univariate comparison between CE group and non-CE group.

\begin{tabular}{|c|c|c|c|}
\hline & CE $(n=97)$ & Non-CE $(n=90)$ & $p$-Value \\
\hline Age, years & $75(66-80)$ & $69(57-79)$ & 0.008 \\
\hline Female, $n(\%)$ & $50(51.5)$ & $25(27.8)$ & 0.001 \\
\hline Smoking, n (\%) & $24(24.7)$ & $40(44.4)$ & 0.005 \\
\hline Hypertension, $n(\%)$ & $71(73.2)$ & $54(60.0)$ & 0.055 \\
\hline Diabetes, $n(\%)$ & $19(19.6)$ & $15(16.7)$ & 0.605 \\
\hline Hyperlipidemia, n (\%) & $39(40.2)$ & $41(45.6)$ & 0.460 \\
\hline Atrial fibrillation, $n(\%)^{\mathrm{a}}$ & $81(83.5)$ & $14(15.6)$ & $<0.001$ \\
\hline Serum glucose, mg/mL & $6.9(6.1-8.2)$ & $6.8(5.9-8.2)$ & 0.460 \\
\hline Platelet counts, $\times 10^{9} / \mathrm{L}$ & 167 (135-199) & 196 (167-229) & $<0.001$ \\
\hline Leukocyte counts, $\times 10^{9} / \mathrm{L}$ & $7.0(5.8-8.7)$ & $8.7(6.9-11.4)$ & $<0.001$ \\
\hline Baseline NIHSS score & $12(8-16)$ & $12(5-16)$ & 0.181 \\
\hline Baseline infarct volume, $\mathrm{mL}$ & $37.2(19.6-65.4)$ & 24.5 (11.9-62.6) & 0.122 \\
\hline $\begin{array}{l}\text { Baseline hypoperfusion } \\
\text { volume, } \mathrm{mL}\end{array}$ & $87.2(48.2-134.2)$ & $84.3(32.6-154.7)$ & 0.79 \\
\hline Baseline mismatch ratio & $2.3(1.7-3.2)$ & $2.5(1.9-3.9)$ & 0.033 \\
\hline $\mathrm{CBF}_{\text {hypo }}, \mathrm{ml} / \mathrm{min} / 100 \mathrm{~g}$ & $6.22(4.61-8.37)$ & $7.21(5.33-9.44)$ & 0.032 \\
\hline rPS hypo, \% & 75.90 (48.39-126.03) & $55.60(26.89-82.57)$ & 0.001 \\
\hline $\mathrm{rPS}_{\text {hypo }}>86.71 \%, n(\%)$ & $43(44.3)$ & $18(20)$ & 0.00 \\
\hline $\mathrm{HT}, n(\%)$ & $46(47.4)$ & $29(32.2)$ & 0.03 \\
\hline $\mathrm{HI}, n(\%)$ & 35 (36.1) & $21(23.3)$ & 0.07 \\
\hline $\mathrm{PH}, n(\%)$ & $11(11.3)$ & 8 (8.9) & 0.63 \\
\hline
\end{tabular}

$\mathrm{CBF}_{\text {hypo, }}$ cerebral blood flow in hypoperfusion region; $\mathrm{CE}$, cardioembolism; HI, hemorrhagic infarction; HT, hemorrhagic transformation; NIHSS, national institute of health stroke scale; $P H$, parenchymal hematoma; $r P S_{\text {hypo, }} r P S$ in hypoperfusion region.

aAtrial fibrillation was not included in the binary logistic regression model. analysis and demonstrated that only female, platelet counts, $\mathrm{rPS}_{\text {hypo }}$ as well as $\mathrm{rPS}_{\text {hypo }}>86.71 \%$ were independently associated with CE (all $p<0.05)$.

\section{DISCUSSION}

To the best of our knowledge, this is the first study to evaluate the association between quantitatively assessed BBBP and stroke subtypes at hyperacute stage. We found that patients with CE had significantly higher baseline PPS $_{\text {hypo }}$ than those with other stroke subtypes.

Previously, a qualitative study found CE was independently associated with BBB disruption, which was defined as the presence of an intraparenchymal hyperdense area exhibited on non-enhanced CT immediately after endovascular therapy (18). In our study, we quantitatively assessed BBBP on PS maps generated from pretreatment CTP. And we found that quantitative BBBP on pretreatment CTP was also associated with stroke subtype.

BBB permeability is elevated in hypoperfusion region as a result of loss of CBF, oxygen, and nutrients (19), while collateral circulation is thought to ameliorate the consequence of the above events (20). Previous study found that less elevated BBBP was associated with more than 50\% collateral filling (21). Cardioembolic occlusion occurs abruptly, and its collateral circulation is thought to be insufficient, leading to more severe hypoperfusion, and therefore higher BBBP compared with LAA $(22,23)$. Interestingly, in the current study, there was no significant difference in either infarct volume or hypoperfusion volume between CE group and non-CE group. It may be partially due to the exclusion of patients with hypoperfusion volume $\leq 10 \mathrm{ml}$. We think the sudden occlusion of artery and lack of collateral circulation may be the major reason of high BBBP in CE. Besides,

TABLE 2 | Multivariable logistic regression analysis for cardioembolism.

\begin{tabular}{|c|c|c|c|}
\hline & Odds ratios & $\begin{array}{c}95 \% \text { confidence } \\
\text { interval }\end{array}$ & $p$-Value \\
\hline \multicolumn{4}{|l|}{ Model 1} \\
\hline Age, years & 1.015 & $0.988-1.043$ & 0.290 \\
\hline Female & 4.026 & $1.682-9.637$ & 0.002 \\
\hline Smoking & 0.971 & $0.419-2.253$ & 0.946 \\
\hline Platelet counts, $\times 10^{9} / \mathrm{L}$ & 0.988 & $0.981-0.995$ & 0.001 \\
\hline Leukocyte counts, $\times 10^{9} / \mathrm{L}$ & 0.894 & $0.779-1.025$ & 0.109 \\
\hline Baseline mismatch ratio & 1.017 & $0.932-1.109$ & 0.710 \\
\hline $\mathrm{CBF}_{\text {hypo }}, \mathrm{ml} / \mathrm{min} / 100 \mathrm{~g}$ & 0.949 & $0.825-1.091$ & 0.462 \\
\hline rPS & 1.008 & $1.001-1.014$ & 0.029 \\
\hline \multicolumn{4}{|l|}{ Model 2} \\
\hline Age, years & 1.013 & $0.985-1.041$ & 0.369 \\
\hline Female & 4.056 & $1.683-9.775$ & 0.002 \\
\hline Smoking & 1.043 & $0.444-2.450$ & 0.924 \\
\hline Platelet counts, $\times 10^{9} / \mathrm{L}$ & 0.987 & $0.979-0.994$ & 0.001 \\
\hline Leukocyte counts, $\times 10^{9} / \mathrm{L}$ & 0.889 & $0.773-1.021$ & 0.095 \\
\hline Baseline mismatch ratio & 1.026 & $0.939-1.211$ & 0.569 \\
\hline $\mathrm{CBF}_{\text {hypo }}, \mathrm{ml} / \mathrm{min} / 100 \mathrm{~g}$ & 0.964 & $0.840-1.106$ & 0.604 \\
\hline rPS hypo $>86.71 \%$ & 3.754 & $1.610-8.753$ & 0.002 \\
\hline
\end{tabular}

$\mathrm{CBF}_{\text {hypo, }}$ cerebral blood flow in hypoperfusion region; rPS hypo, rPS in hypoperfusion region. 
Olivot et al. found that endogenous tissue-type plasminogen activator (t-PA) was increased in CE (24). Previous study also found patients with atrial fibrillation, regardless of their history of ischemic stroke, had higher endogenous t-PA levels than patients in sinus rhythm (25). Set aside its thrombolytic effect, t-PA could be toxic to ischemic brain tissue, including BBB, through t-PA-dependent pathways and plasmin-dependent pathways $(26,27)$. This can be another evidence that links $\mathrm{BBB}$ disruption and $\mathrm{CE}$. Moreover, previous studies revealed that C-reactive protein (CRP) was higher in patients with $\mathrm{CE}$ $(28,29)$. It has been proved that CRP could cause endothelial cells contraction, which went along with disruption of tight junction molecules and finally resulting in BBB disruption (30). Matrix metalloproteinase 9, which could disrupt BBB through degradation of extracellular matrix components of the basal lamina, was also increased after CE stroke (31). All of these pieces of evidence (including ours) indicate that CE might be associated with increased BBBP.

Our finding about the hyper-permeability of $\mathrm{BBB}$ in $\mathrm{CE}$ patients may suggest that the application of $\mathrm{BBB}$ protecting drugs is helpful especially for CE patients in the future. Interestingly, a recent study found that $\mathrm{BBB}$ disruption was reversible after reperfusion (32), although high baseline BBBP might lead to HT and malignant edema (33). Moreover, the increase of reperfusion rate was positively associated with the decrease of BBBP (32). Therefore, reperfusion therapy would still be a good choice for CE patients at acute stage. As we mentioned before, CE patients had more endogenous t-PA that might be harmful to $\mathrm{BBB}$. Nevertheless, considering the benefit of recanalization, intravenous recombinant t-PA should still be the first aid for CE within time window according to the guideline.

Our study had several limitations. First, the retrospective design might have a potential risk of selection bias. All patients in this study received IVT. Because treatment should not affect pretreatment BBBP as well as stroke subtype, the results should not be affected by the selected patients. About $10 \%$ patients in our study were excluded because of inadequate image quality for the assessment of PS. The measurement of PS is based on arterial input function (AIF) curve and venous output function (OVF) curve. Most defective AIF and OVF curve are due to excessive motion during imaging and trouble in contrast injection, which are unavoidable in practice. Second, patients with hypoperfusion volume less than $10 \mathrm{ml}$ were not included in this study. One reason is for its low signal-to-noise ratio; another is that when drawing ROIs in such small areas, relatively larger manual measurement error may be introduced. Third, the CTP measurement technique used in this study was first-pass with a short acquisition time (74.5 s). Previous studies suggested that it was better to use BBBP estimated from the delayed phase $(34,35)$. However, there was a linear relationship between BBBP measured from first-pass and delayed phase (36). In addition, the delayed phase extends the acquisition time to at least $210 \mathrm{~s}$, which may further increase the radiation dose and risk of motion artifacts. Our sequence is much more practical and generalizable to everyday clinical practice. Fourth, there were 14 patients had atrial fibrillation in non-CE group and all of them had stenosis of ipsilateral major cerebral artery over $50 \%$. We could not exclude the role of atrial fibrillation in infarct among these patients, which might lead to the underestimate of the difference of BBBP between CE group and non-CE group.

In conclusion, the current study demonstrated that BBBP in the hypoperfusion region was higher in CE patients than other stroke subtypes, indicating severe BBB disruption in cardioembolic stroke. Further prospective studies are needed to confirm this finding.

\section{ETHICS STATEMENT}

All subjects had given written informed consent prior to the study, and the protocols had been approved by the human ethics committee of the Second Affiliated Hospital of Zhejiang University School of Medicine. All clinical investigations have been conducted according to the principles expressed in the Declaration of Helsinki.

\section{AUTHOR CONTRIBUTIONS}

CL drafted and revised the manuscript, participated in study concept and design, conducted the statistical analyses, analyzed, and interpreted the data. ML participated in study concept and design, data interpretation, and made a major contribution in revising the manuscript. FS, ZC, and SY participated in the study design and made contribution in revising the manuscript. CL, FS, and XD participated in the data acquisition and analysis.

\section{ACKNOWLEDGMENTS}

The perfusion analysis software (MIStar) was provided to the site as part of their involvement in the International Stroke Perfusion Imaging Registry (INSPIRE, www.Inspire.apollomit. $\mathrm{com} /$ ), study funded by the National Health and Medical Research Council of Australia.

\section{FUNDING}

This work was supported by the National Natural Science Foundation of China (81471170 \& 81622017 \& 81601017) and the Science Technology Department of Zhejiang Province (2017C04G1360592).

\section{SUPPLEMENTARY MATERIAL}

The Supplementary Material for this article can be found online at https://www.frontiersin.org/articles/10.3389/fneur.2018.00055/ full\#supplementary-material. 


\section{REFERENCES}

1. Doufekias E, Segal AZ, Kizer JR. Cardiogenic and aortogenic brain embolism. J Am Coll Cardiol (2008) 51(11):1049-59. doi:10.1016/j.jacc.2007.11.053

2. Paciaroni M, Agnelli G, Corea F, Ageno W, Alberti A, Lanari A, et al. Early hemorrhagic transformation of brain infarction: rate, predictive factors, and influence on clinical outcome: results of a prospective multicenter study. Stroke (2008) 39(8):2249-56. doi:10.1161/STROKEAHA.107.510321

3. England TJ, Bath PM, Sare GM, Geeganage C, Moulin T, O’Neill D, et al. Asymptomatic hemorrhagic transformation of infarction and its relationship with functional outcome and stroke subtype: assessment from the tinzaparin in acute ischaemic stroke trial. Stroke (2010) 41(12):2834-9. doi:10.1161/ STROKEAHA.109.573063

4. Latour LL, Kang DW, Ezzeddine MA, Chalela JA, Warach S. Early bloodbrain barrier disruption in human focal brain ischemia. Ann Neurol (2004) 56(4):468-77. doi:10.1002/ana.20199

5. Leigh R, Jen SS, Hillis AE, Krakauer JW, Barker PB, STIR and VISTA Imaging Investigators. Pretreatment blood-brain barrier damage and post-treatment intracranial hemorrhage in patients receiving intravenous tissue-type plasminogen activator. Stroke (2014) 45(7):2030-5. doi:10.1161/ STROKEAHA.114.005249

6. Jauch EC, Saver JL, Adams HP Jr, Bruno A, Connors JJ, Demaerschalk BM, et al. Guidelines for the early management of patients with acute ischemic stroke: a guideline for healthcare professionals from the American Heart Association/American Stroke Association. Stroke (2013) 44(3):870-947. doi:10.1161/STR.0b013e318284056a

7. Adams HP Jr, Bendixen BH, Kappelle LJ, Biller J, Love BB, Gordon DL, et al. Classification of subtype of acute ischemic stroke. Definitions for use in a multicenter clinical trial. TOAST. Trial of Org 10172 in acute stroke treatment. Stroke (1993) 24(1):35-41. doi:10.1161/01.STR.24.1.35

8. St Lawrence KS, Lee TY. An adiabatic approximation to the tissue homogeneity model for water exchange in the brain: I. Theoretical derivation. J Cereb Blood Flow Metab (1998) 18(12):1365-77. doi:10.1097/00004647-199812000-00011

9. Koh TS, Cheong LH, Tan CK, Lim CC. A distributed parameter model of cerebral blood-tissue exchange with account of capillary transit time distribution. Neuroimage (2006) 30(2):426-35. doi:10.1016/j.neuroimage.2005.09.032

10. Aviv RI, d'Esterre CD, Murphy BD, Hopyan JJ, Buck B, Mallia G, et al. Hemorrhagic transformation of ischemic stroke: prediction with CT perfusion. Radiology (2009) 250(3):867-77. doi:10.1148/radiol.2503080257

11. Lin L, Bivard A, Levi CR, Parsons MW. Comparison of computed tomographic and magnetic resonance perfusion measurements in acute ischemic stroke: back-to-back quantitative analysis. Stroke (2014) 45(6):1727-32. doi:10.1161/ STROKEAHA.114.005419

12. Campbell BC, Christensen S, Levi CR, Desmond PM, Donnan GA, Davis SM, et al. Cerebral blood flow is the optimal CT perfusion parameter for assessing infarct core. Stroke (2011) 42(12):3435-40. doi:10.1161/STROKEAHA. 111.618355

13. Campbell BC, Mitchell PJ, Yan B, Parsons MW, Christensen S, Churilov L, et al. A multicenter, randomized, controlled study to investigate EXtending the time for Thrombolysis in Emergency Neurological Deficits with Intra-Arterial therapy (EXTEND-IA). Int J Stroke (2014) 9(1):126-32. doi:10.1111/ijs.12206

14. Dankbaar JW, Hom J, Schneider T, Cheng SC, Lau BC, van der Schaaf I, et al. Age- and anatomy-related values of blood-brain barrier permeability measured by perfusion-CT in non-stroke patients. JNeuroradiol (2009) 36(4):219-27. doi:10.1016/j.neurad.2009.01.001

15. Horsch AD, Dankbaar JW, van Seeters T, Niesten JM, Luitse MJ, Vos PC, et al. Relation between stroke severity, patient characteristics and CT-perfusion derived blood-brain barrier permeability measurements in acute ischemic stroke. Clin Neuroradiol (2015) 26(4):415-21. doi:10.1007/s00062-015-0375-1

16. Hacke W, Kaste M, Fieschi C, Toni D, Lesaffre E, von Kummer R, et al. Intravenous thrombolysis with recombinant tissue plasminogen activator for acute hemispheric stroke. The European Cooperative Acute Stroke Study (ECASS). JAMA (1995) 274(13):1017-25. doi:10.1001/jama.1995. 03530130023023

17. Amarenco P, Bogousslavsky J, Caplan LR, Donnan GA, Wolf ME, Hennerici MG. The ASCOD phenotyping of ischemic stroke (Updated ASCO Phenotyping). Cerebrovasc Dis (2013) 36(1):1-5. doi:10.1159/000352050

18. Desilles JP, Rouchaud A, Labreuche J, Meseguer E, Laissy JP, Serfaty JM, et al. Blood-brain barrier disruption is associated with increased mortality after endovascular therapy. Neurology (2013) 80(9):844-51. doi:10.1212/ WNL.0b013e31828406de

19. Sandoval KE, Witt KA. Blood-brain barrier tight junction permeability and ischemic stroke. Neurobiol Dis (2008) 32(2):200-19. doi:10.1016/j. nbd.2008.08.005

20. Bang OY, Saver JL, Alger JR, Starkman S, Ovbiagele B, Liebeskind DS, et al. Determinants of the distribution and severity of hypoperfusion in patients with ischemic stroke. Neurology (2008) 71(22):1804-11. doi:10.1212/01. wnl.0000335929.06390.d3

21. Dankbaar JW, Hom J, Schneider T, Cheng SC, Bredno J, Lau BC, et al. Dynamic perfusion-CT assessment of early changes in blood brain barrier permeability of acute ischaemic stroke patients. J Neuroradiol (2011) 38(3):161-6. doi:10.1016/j.neurad.2010.08.001

22. Jung JM, Kwon SU, Lee JH, Kang DW. Difference in infarct volume and patterns between cardioembolism and internal carotid artery disease: focus on the degree of cardioembolic risk and carotid stenosis. Cerebrovasc Dis (2010) 29(5):490-6. doi:10.1159/000297965

23. Rebello LC, Bouslama M, Haussen DC, Grossberg JA, Dehkharghani S, Anderson A, et al. Stroke etiology and collaterals: atheroembolic strokes have greater collateral recruitment than cardioembolic strokes. Eur J Neurol (2017) 24(6):762-7. doi:10.1111/ene.13287

24. Olivot JM, Labreuche J, Mahagne MH, Aiach M, Amarenco P, Investigators G. Endogenous tissue-type plasminogen activator and cardioembolic brain infarct subtype. J Thromb Haemost (2006) 4(11):2513-4. doi:10.1111/j.1538-7836.2006.02170.x

25. Kahn SR, Solymoss S, Flegel KM. Increased tissue plasminogen activator levels in patients with nonvalvular atrial fibrillation. CMAJ (1997) 157(6):685-9.

26. Wang X, Tsuji K, Lee SR, Ning M, Furie KL, Buchan AM, et al. Mechanisms of hemorrhagic transformation after tissue plasminogen activator reperfusion therapy for ischemic stroke. Stroke (2004) 35(11 Suppl 1):2726-30. doi:10.1161/01.STR.0000143219.16695.af

27. Niego B, Medcalf RL. Plasmin-dependent modulation of the blood-brain barrier: a major consideration during tPA-induced thrombolysis? J Cereb Blood Flow Metab (2014) 34(8):1283-96. doi:10.1038/jcbfm.2014.99

28. Chaudhuri JR, Mridula KR, Umamahesh M, Swathi A, Balaraju B, Bandaru VC. High sensitivity C-reactive protein levels in acute ischemic stroke and subtypes: a study from a tertiary care center. Iran J Neurol (2013) 12(3):92-7.

29. Lehmann MF, Kallaur AP, Oliveira SR, Alfieri DF, Delongui F, de Sousa Parreira J, et al. Inflammatory and metabolic markers and short-time outcome in patients with acute ischemic stroke in relation to TOAST subtypes. Metab Brain Dis (2015) 30(6):1417-28. doi:10.1007/s11011-015-9731-8

30. Kuhlmann CR, Librizzi L, Closhen D, Pflanzner T, Lessmann V, Pietrzik $\mathrm{CU}$, et al. Mechanisms of C-reactive protein-induced blood-brain barrier disruption. Stroke (2009) 40(4):1458-66. doi:10.1161/STROKEAHA.108. 535930

31. Montaner J, Alvarez-Sabin J, Molina CA, Angles A, Abilleira S, Arenillas J, et al. Matrix metalloproteinase expression is related to hemorrhagic transformation after cardioembolic stroke. Stroke (2001) 32(12):2762-7. doi:10.1161/ hs1201.99512

32. Simpkins AN, Dias C, Leigh R; National Institutes of Health Natural History of Stroke Investigators. Identification of reversible disruption of the human blood-brain barrier following acute ischemia. Stroke (2016) 47(9):2405-8 doi:10.1161/STROKEAHA.116.013805

33. Hom J, Dankbaar JW, Soares BP, Schneider T, Cheng SC, Bredno J, et al. Blood-brain barrier permeability assessed by perfusion CT predicts symptomatic hemorrhagic transformation and malignant edema in acute ischemic stroke. AJNR Am J Neuroradiol (2011) 32(1):41-8. doi:10.3174/ajnr. A2244

34. Dankbaar JW, Hom J, Schneider T, Cheng SC, Lau BC, van der Schaaf I, et al. Dynamic perfusion CT assessment of the blood-brain barrier permeability: first pass versus delayed acquisition. AJNR Am J Neuroradiol (2008) 29(9):1671-6. doi:10.3174/ajnr.A1203

35. Hom J, Dankbaar JW, Schneider T, Cheng SC, Bredno J, Wintermark M. Optimal duration of acquisition for dynamic perfusion CT assessment of blood-brain barrier permeability using the patlak model. AJNR Am J Neuroradiol (2009) 30(7):1366-70. doi:10.3174/ajnr.A1592

36. Nguyen GT, Coulthard A, Wong A, Sheikh N, Henderson R, O'Sullivan JD, et al. Measurement of blood-brain barrier permeability in acute ischemic 
stroke using standard first-pass perfusion CT data. Neuroimage Clin (2013) 2:658-62. doi:10.1016/j.nicl.2013.04.004

Conflict of Interest Statement: The authors declare that the research was conducted in the absence of any commercial or financial relationships that could be construed as a potential conflict of interest.
Copyright $\odot 2018 \mathrm{Liu}$, Shi, Chen, Yan, Ding and Lou. This is an open-access article distributed under the terms of the Creative Commons Attribution License (CC BY). The use, distribution or reproduction in other forums is permitted, provided the original author(s) and the copyright owner are credited and that the original publication in this journal is cited, in accordance with accepted academic practice. No use, distribution or reproduction is permitted which does not comply with these terms. 